\title{
Synthesis of Dual-Beam Patterns by Exploiting Time- Modulation in Unequally Spaced Linear Arrays
}

\author{
Somnath PATRA ${ }^{1,2}$, Sujit Kumar MANDAL ${ }^{2}$, Gautam Kumar MAHANTI ${ }^{2}$, Narendra Nath PATHAK ${ }^{3}$ \\ ${ }^{1}$ Dept. of Electronics and Communication Engineering, SMIT (TIG), Guptipara, Hooghly, WB, India \\ ${ }^{2}$ Dept. of Electronics and Communication Engineering, NIT Durgapur, WB, India \\ ${ }^{3}$ Dept. of Electronics and Communication Engineering, Dr. B. C. Roy Engineering College, Durgapur, WB, India
}

somnath.tech@gmail.com, skmanda12006@gmail.com,gkm@ece.nitdgp.ac.in, narendra.pathak@bcrec.ac.in

Submitted May 17, 2021 / Accepted October 22, 2021

\begin{abstract}
In this paper, a novel approach for synthesizing multiple radiation patterns with reduced hardware complexity in the feed network by exploiting the additional degree of freedom 'time' in time modulated unequally spaced linear array (TMUSLA) is presented. In the proposed approach, with a suitable common set of element position of TMUSLA, the desired dual-beam pattern with low sidelobe level (SLL) is obtained by simply controlling the ON-OFF time sequence of the RF switches connected to the array elements. To show the effectiveness of the proposed array synthesis method, two dual beam patterns first one as pencil (sum) beam (PB) and flat-topped beam (FTB) pattern, and the second one as sum and difference pattern with different constraints have been synthesized. For the successful generation of the desired power patterns, differential evolution (DE) algorithm is employed to obtain the optimum possible solution in terms of common element position, time-modulation, switching sequences and applicable excitation phase for the desired shape beam patterns. The superiority of the proposed approach with the favourable improved performance has been demonstrated by comparing the realized patterns with the stateof-the-art relevant reported works.
\end{abstract}

\section{Keywords}

Pattern synthesis, on-time duration, side lobe level, sideband level, differential evolution

\section{Introduction}

In many applications including wireless communication [1], radar systems [2] and terrestrial or vehicular systems, multiple dual-beam patterns have been widely used due to their requirement of limited space in antenna installation. In conventional phased array (CPA), for a particular pattern, the satisfactory excitation amplitudes, phases and positions of the elements are evaluated such that the radiation pattern produced by the array can be approximated closed to the desired one [3-5]. For a set of desired multi- ple patterns, suitable switching devices are utilized to select the predetermined excitations of the corresponding patterns [6]. However, for each pattern, a set of attenuators and phase shifters is used in the feed network of the array. This increases the overall cost, weight and complexity to synthesize multiple radiation patterns in CPA system. Further, some desired patterns with the stringent requirement of low SLLs cannot be realizable due to the practical limitations of unwanted error of tolerance to provide the proper excitation amplitude and phase. In this framework, over the past decade, time modulated arrays (TMAs) have been used. In TMA, the radiation performance of the antenna elements are controlled by periodic on-off sequences of a set of RF switches that modulates the static excitation amplitude and phase array weights using "time" as an additional degree of freedom. The time-modulation technique as introduced by Kummar et al. [7], was first employed to synthesize low SLL patterns with uniform or reduced dynamic range ratio (DRR) of static excitation amplitudes [8]. During past 60 years, various array pattern synthesis methods have been presented, such as Taylor-Kaiser [9], Dolph Chebyshev [10]. However, these analytical methods are used to generate element excitation for synthesizing pencil beam patterns in equally spaced array. On the other hand, unequally spaced antenna array has the advantage of providing low sidelobe pattern with uniform static excitation amplitude and phase. The major problem in designing unequally spaced array is that in addition to the excitation amplitude and phase, inclusion of the element spacing as another optimization parameter leads to increase the problem dimension. Different pattern synthesis methods based on convex optimization (CVX) [11], [12], iterative FFT [13] have been successfully applied to synthesize the desired patterns by finding out the appropriate antenna array design parameters. In the past two decades, time-modulation principle is emerged as one potential method to synthesize antenna array patterns [14-17]. Towards this, many nature inspired global optimization algorithms such as differential evolution (DE) [14], [15], simulated annealing (SA) [16], genetic algorithm (GA) [17], particle swarm optimization (PSO) [18] are used. Also, some methods based on different time-modulation techniques have been 
employed in this regards [19], [20]. Further, the advantage of using the additional degree of freedom namely, 'time' is applied to synthesize shaped beam patterns [21], [22], dual-beam sum and difference patterns [23], dual-beam harmonic patterns [24] and multiple power patterns [25]. Although numerous methods have been proposed to synthesize equally spaced time-modulated arrays, the multiple pattern synthesis problem using time-modulation in unequally spaced antenna array is rarely considered [26]. In [26], the time-modulation principle is used to synthesize low SLL pencil beam pattern in unequally spaced antenna array. In this article, the potentiality of time-modulation is employed to synthesize different dual-beam patterns, specifically, pencil-flat topped and sum-difference, in unequally spaced linear array. Without large number of phase shifters and attenuators as required to synthesize such patterns in CPAs, the desired patterns have been realized by controlling the ON-OFF status of the switches by using a simple, cost-effective switching circuit in the feed network. The efficient global search optimization algorithm, namely, DE is employed to find out the unknown array design parameters of the desired patterns. By comparing the results of state-of-the-art relevant literatures, it is seen that time modulated linear arrays (TMLAs) with unequally spaced elements are more capable of synthesizing the desired patterns of low/ultra-low SLL.

The rest of the paper is organized as follows. Theoretical analysis and problem formulation has been discussed in Sec. 2. Numerical result to show the effectiveness of the proposed approach is presented in Sec. 3. Finally, some conclusion about the work has been written in Sec. 4.

\section{Theoretical Analysis and Problem Formulation}

\subsection{Theory}

Let us consider an unequally spaced, symmetric linear array with $2 N$ number of isotropic radiators placed along the $\mathrm{x}$-axis. For the positive half of the array structure as shown in Fig. 1, the locations of the individual antenna elements are represented as, $\mathbf{X}=\left\{x_{1}, x_{2}, \ldots, x_{N}\right\}$ while the negative half is the mirror image of the positive half. If each of the element in the array is connected with highspeed RF switches, then the array factor $[F(\theta, t)]$, of such array [19], [27] is expressed as

$$
F(\theta, \mathrm{t})=E_{\mathrm{f}}(\theta) \mathrm{e}^{\mathrm{j} \omega_{0} t} \sum_{\substack{n=-N \\ n \neq 0}}^{N} A_{n} \mathrm{e}^{\mathrm{j} \alpha_{n}} U_{n}(t) \cdot \mathrm{e}^{\mathrm{j} \beta x_{n} \cos \theta}
$$

where $E_{\mathrm{f}}(\theta)$ and $\omega_{0}=2 \pi f_{0}$ are denoted as element factor and operating angular frequency, respectively. $A_{n}$ and $\alpha_{n}$ are the static excitation amplitude and phase. $x_{n}$ is the location of the $n^{\text {th }}$ element from the reference position taken as

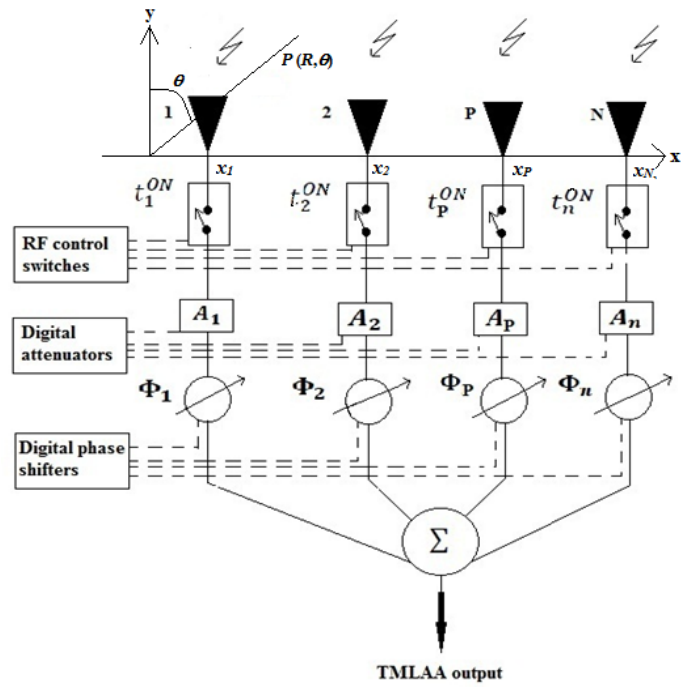

Fig. 1. A typical block diagram of the positive half of a symmetrically placed $2 \mathrm{~N}$ - element time-modulated unequally spaced linear array geometry.

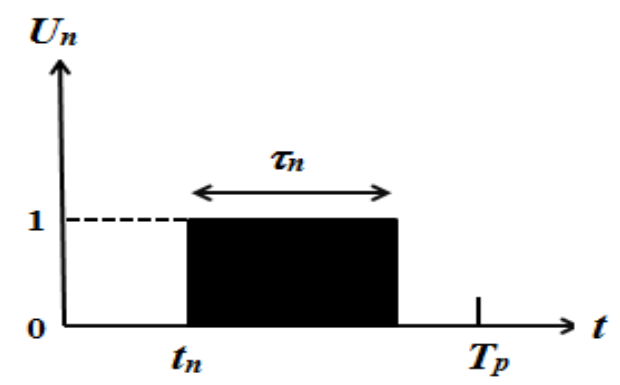

Fig. 2. Schematic of time-switching function.

array center, coinciding with the origin of the coordinate system; $\beta=2 \pi f_{0} / c$ is the free-space wave number, $c$ be the velocity of the light in free space; $\theta$ is the angle measured from the broadside direction of the array and $U_{n}(t)$ is the time switching function with time period $T_{\mathrm{p}}$, given by,

$$
U_{n}(\mathrm{t})=\left\{\begin{array}{lc}
1, & t_{n} \leq t \leq t_{n}+\tau_{n} \\
0, & \text { otherwise }
\end{array}\right.
$$

where $t_{n}$ is the ON-time instant (OTI), and $\tau_{n}$ is the ONtime duration (OTD) of the $n^{\text {th }}$ element as shown in Fig. 2. Due to the periodicity of $U_{n}(t)$, its Fourier representation turns out to be

$$
U_{n}(t)=\sum_{h=-\infty}^{\infty} u_{h n} \exp \left(\mathrm{j} h \omega_{\mathrm{p}} t\right)
$$

where $\omega_{\mathrm{p}}=2 \pi f_{\mathrm{p}}=2 \pi / T_{\mathrm{p}}$ is the modulation frequency in $\mathrm{rad} / \mathrm{sec}$ and $h$ is the harmonic number. The Fourier coefficient at the $h^{\text {th }}$ harmonic order due to periodical switching of the $n^{\text {th }}$ antenna element, $u_{h n}$ is given by

$$
u_{h n}=\frac{1}{T_{\mathrm{p}}} \int_{0}^{T_{\mathrm{p}}} U_{n}(t) \exp \left(-\mathrm{j} h \omega_{\mathrm{p}} t\right) \mathrm{d} t .
$$

By considering isotropic radiators [i.e. $E_{\mathrm{f}}(\theta)=1$ ], the far-field radiation pattern can be represented as [16] 


$$
\begin{aligned}
F(\theta, t) & =\sum_{h=-\infty}^{\infty}\left[\sum_{\substack{n=-N \\
n \neq 0}}^{N} A_{h n} \mathrm{e}^{\mathrm{j}\left(\beta x_{n} \cos \theta+\alpha_{n}\right)}\right] \mathrm{e}^{\mathrm{j}\left(\mathrm{h} \omega_{\mathrm{p}}+\omega_{0}\right) t} \\
& =\sum_{h=-\infty}^{\infty} F_{h}(\theta, t)
\end{aligned}
$$

where $A_{h n}=A_{n} u_{h n}$, and $F_{h}(\theta, t)$ is the array factor at the $h^{\text {th }}$ harmonic and is given as

$$
F_{h}(\theta, t)=\left[\sum_{\substack{n=-N \\ n \neq 0}}^{N} A_{h n} \mathrm{e}^{\mathrm{j}\left(\beta x_{n} \cos \theta+\alpha_{n}\right)}\right] \mathrm{e}^{\mathrm{j}\left(h \omega_{\mathrm{p}}+\omega_{0}\right) t} .
$$

The beam pattern at the operating carrier frequency $f_{0}$ depends upon the $0^{\text {th }}$ order coefficients and is given as

$$
A_{0 n}=A_{n} u_{0 n} ; \forall n \in\{-N, N\} \text {. }
$$

In (7), $u_{0 n}$ is the $0^{\text {th }}$ order Fourier coefficient and is obtained as

$$
u_{0 n}=\frac{1}{T_{\mathrm{p}}} \int_{0}^{T_{\mathrm{p}}} U_{n}(t) \mathrm{d} t=\xi_{n}
$$

where $\xi_{n}=\tau_{n} / T_{\mathrm{p}}$ is the normalized OTD. However, the radiation at different sideband depends on the non-zero harmonic order of the Fourier coefficient $u_{h n}(h \neq 0)$ and is given as

$$
u_{h n}=A_{n} \xi_{n} \frac{\sin \left(h \pi \xi_{n}\right)}{h \pi \xi_{n}} \mathrm{e}^{-\mathrm{j} h \pi\left(2 \sigma_{n}+\xi_{n}\right)}
$$

where $\sigma_{n}=t_{n} / T_{\mathrm{p}}$ is the normalized OTI of the switch. Equations (6)-(9) indicate that radiation pattern at center frequency $(h=0)$ depends only on the normalized OTD $\xi_{n} ; \forall n \in\{-N, N\}$ while the signal radiated at different sideband is governed by both OTD and OTI, $\xi_{n}$ and $\sigma_{n} ; \forall n \in\{-N, N\}$. Thus, for the time-modulated unequally spaced linear array (TMUSLA), the desired power patterns at the center frequency can be synthesized with proper selection of the element positions and OTDs while both OTDs and OTIs of the elements can be tuned to suppress the undesired sideband radiations. However, for the multipattern application of TMUSLA, if different pattern is synthesized with different set of element position, it will be practically difficult to switch from one pattern to the other by changing their respective element positions. Thanks to the time-modulation technique where a common set of element position can be fixed for the desired multi-beam patterns while the individual pattern differs with OTDs and OTIs of the switches connected to the array elements. Thus, switching from one pattern to the other pattern of the array can be achieved easily, electronically by controlling the on-off status of the switches with their respective predetermined OTDs and OTIs with the help of a computer program.

\subsection{Problem Formulation}

DE algorithm is one of the efficient nature inspired global optimization algorithm, due to its efficient search ability and fast convergence rate. DE is also successfully applied to many antennas [22-27], electromagnetics [28] as well as many other optimization problems. To synthesize the desired dual-beam patterns at the center frequency by suppressing the sideband radiations, the cost function to be minimized using DE is defined as,

$$
\psi=\sum_{\substack{b=\{F, S\} \\ \text { or } b=\{S, D\}}}\left\{\sum_{a=1}^{3} W_{a}^{b} \delta_{a}^{b} \mathrm{H}_{a}\left(\delta_{a}^{b}\right)\right\}
$$

where $F, S$, and $D$ in (10) stand for flat-top, sum (pencil), and difference pattern. $\delta_{a}^{b}$ with $a=1,2$ and 3 represents the difference between desired and obtained values of the design parameters of the respective beam and are given as follows. For all the individual patterns, $\delta_{1}{ }^{b}$ and $\delta_{2}{ }^{b}$ are defined as $\delta_{1}^{b}=\left|S L L_{\mathrm{d}}^{b}-S L L_{\text {max }}^{b}\right|, \quad \delta_{2}^{b}=\left|S B L_{\mathrm{d}}^{b}-S B L_{\text {max }}^{b}\right|$. However, for the flat-topped pattern, $\delta_{3}^{b}$ is defined as $\delta_{3}^{b}=\left|\operatorname{Rip}_{\mathrm{d}}{ }^{(b=F)}-\operatorname{Rip}_{\max }{ }^{(b=F)}\right|$ while, for the sum and difference patterns the design parameter is considered as $\delta_{3}^{b}=\left|F N B W_{\mathrm{d}}^{(b=S, D)}-F N B W_{\max }{ }^{(b=S, D)}\right| . \quad$ Here, $S L L_{\max }$ and $S B L_{\max }$ represent the maximum values of sidelobe and sideband level; Rip $p_{\max }$ is the obtained value of the maximum ripple in the flat region of the pattern; $F N B W_{\max }$ is the obtained beam-width between the first null of the main beam; $S L L_{\mathrm{d}}, S B L_{\mathrm{d}}, R i p_{\mathrm{d}}$ and $F N B W_{\mathrm{d}}$ are their respective desired parameters; $W_{a}$ is the weighting factors of the related design parameters and $H_{a}$ stands for Heaviside step function.

\section{Results and Discussion}

In this section few cases are considered and compared with the published results to establish the superiority of the proposed approach.

\subsection{Case 1: Synthesis of Pencil and Flat- Topped Patterns Controlled by OTDs of the Switches}

In this case, a TMLA of 20 isotropic elements is considered for synthesis of a pencil and a flat-topped pattern. The excitation amplitude distribution of the TMLA is pre-fixed as uniform, leaving the on-time duration (OTD) of the elements are different for each pattern. The main objective is to find out a common set of element position for the desired dual patterns while different set of OTDs are responsible to shape the pattern in pencil and flat-topped form. To synthesize the pattern at center frequency the desired values of $S L L, S B L$ and Rip in the cost function are set as $S L L_{\mathrm{d}}=-20 \mathrm{~dB}, S B L_{\mathrm{d}}=-30 \mathrm{~dB}$ and $R i p_{\mathrm{d}}=0.5 \mathrm{~dB}$. The search range of the optimization parameters vectors are defined as follows: excitation phase 
for flat-topped pattern as $(-\pi, \pi)$; common element positions as $(0.5 \lambda, \lambda)$; OTDs for the pencil beam as $\left(0.1 T_{\mathrm{P}}, T_{\mathrm{P}}\right)$ while for flat-topped beam it is defined as $\left(0.05 T_{\mathrm{P}}, T_{\mathrm{P}}\right)$. The controlling parameters of DE algorithm are chosen as population number $N_{\mathrm{p}}=90$, mutation constant $F=0.5$, crossover probability rate $C_{\mathrm{r}}=0.85$. For synthesizing the desired dual patterns from the symmetric 20 element TMLA, the number of unknown parameters to be determined are: common element positions - 10, OTDs corresponding to both PB and FTB - 20 (10 for each) and excitation phase for the FTB - 10. In order to find the optimum solution, DE continuously minimizes the cost function as defined in (10), until it meets the required conditions or reaches to maximum iteration limit of 500. DE optimized on-time durations for dual beam pattern are shown in Fig. 3.

The DE optimized pencil and flat-topped pattern at center frequency $f_{0}$ is depicted in Fig. 4 . The common element position for both PB and FTB and the required excitation phase for the FTB are shown in Fig. 5. For the synthesized dual-beam patterns, the values of SLL and SBL are suppressed as low as to $-24.60 \mathrm{~dB}$ and $-23.92 \mathrm{~dB}$ in case of pencil beam whereas in case of flat-top beam the values are suppressed to $-24.75 \mathrm{~dB}$ and $-14.73 \mathrm{~dB}$ respectively. The obtained value of the maximum ripple Rip $_{\max }$ in the flat region of the flat-topped pattern is 0.45 .

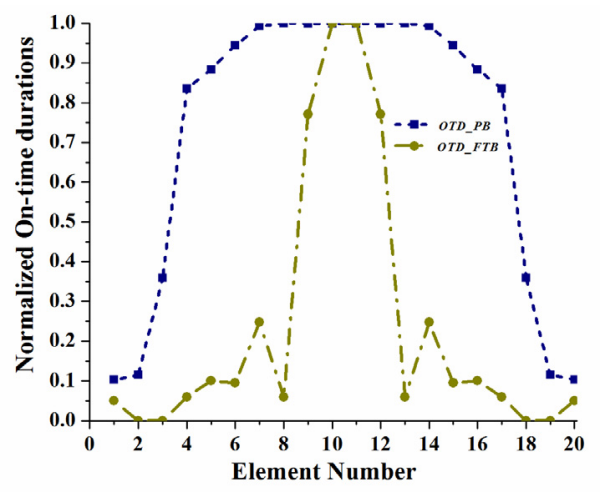

Fig. 3. Graphically represented DE optimized OTDs for pencil beam pattern and flat-top beam pattern.

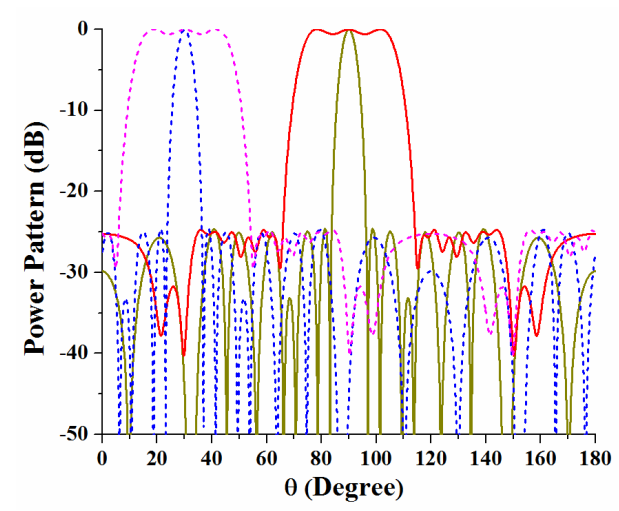

Fig. 4. DE optimized pencil and flat-top shaped pattern of $N=20$, with unequal element spacing where mainlobe is at $\theta=30^{\circ}$ and $\theta=90^{\circ}$.

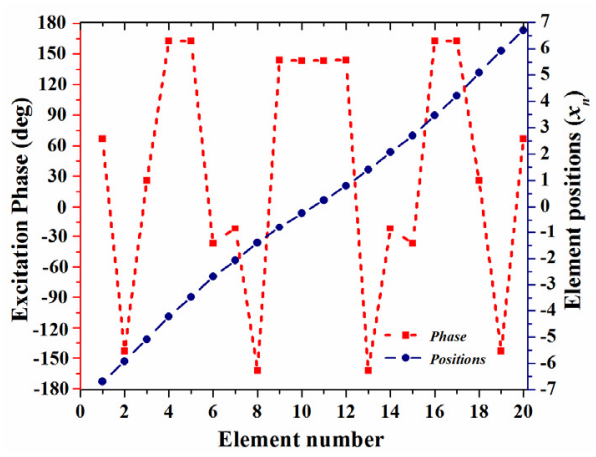

Fig. 5. DE optimized element position and excitation phase obtained for dual beam patterns.

\begin{tabular}{|c|c|c|c|c|c|}
\hline \multirow{3}{*}{ Method } & \multicolumn{3}{|c|}{$\begin{array}{c}\text { Obtained values of design } \\
\text { parameter }\end{array}$} & \multirow{3}{*}{ Array type } & \multirow{3}{*}{$\begin{array}{c}\text { Optimization } \\
\text { parameter vector }\end{array}$} \\
\hline & \multirow{2}{*}{\begin{tabular}{|c|}
$\begin{array}{c}\text { Pencil } \\
\text { beam } \\
\text { (PB) }\end{array}$ \\
$S L L$ \\
(dB)
\end{tabular}} & \multicolumn{2}{|c|}{$\begin{array}{l}\text { Flat-topped } \\
\text { beam (FTB) }\end{array}$} & & \\
\hline & & $\begin{array}{l}S L L \\
(\mathrm{~dB})\end{array}$ & $\begin{array}{l}\operatorname{Rip}_{\max } \\
\text { (dB) }\end{array}$ & & \\
\hline Ref. [25] & -20 & -20 & 0.5 & $\begin{array}{c}\text { UA- } \\
\text { TMESLA }\end{array}$ & $\left\{\tau_{n}, \alpha_{n}\right\}$ \\
\hline Ref. [29] & -30.27 & -25 & 0.5 & $\begin{array}{c}\text { NUA- } \\
\text { CUSLA }^{*}\end{array}$ & $\begin{array}{c}\left\{x_{n}, \alpha_{n}, \text { and } A_{n} \text { with }\right. \\
\text { DRR of } 10.29\}\end{array}$ \\
\hline Ref. [30] & -16 & -10 & 0.5 & UA-CESLA $^{*}$ & $\left\{\alpha_{n}\right\}$ \\
\hline Ref. [31] & -24.13 & -21.17 & 0.47 & NUA-CESLA* & $\begin{array}{c}\left\{\alpha_{n}, \text { and } A_{n} \text { with }\right. \\
\text { DRR of } 4.98\}\end{array}$ \\
\hline Ref. [32] & -20 & -20 & 0.75 & NUA-CESLA* & $\begin{array}{c}\left\{\alpha_{n}, \text { and } A_{n} \text { with }\right. \\
\text { DRR of } 3.5\}\end{array}$ \\
\hline $\begin{array}{l}\text { Proposed } \\
\text { Method }\end{array}$ & -24.60 & -24.75 & 0.45 & $\begin{array}{c}\text { UA- } \\
\text { TMUSLA }\end{array}$ & $\begin{array}{l}\left\{x_{n}, \tau_{n} \text { and } \alpha_{n} \text { only }\right. \\
\text { for shaped beam }\}\end{array}$ \\
\hline
\end{tabular}

Tab. 1. Comparison of design parameters, optimization parameters and antenna types for pencil beam (PB) and flat-top beam (FTB). Note: UA - Uniform Amplitude; NUA - Non-uniform amplitude; TMESLA - timemodulated equally spaced linear array; TMUSLA time-modulated unequally spaced linear array; CESLA - conventional equally spaced linear array; CUSLA conventional unequally spaced linear array.

Table 1 compares different parameters of the dual beam patterns obtained under the proposed method and other related reported results. In [25], for an equally $(\lambda / 2)$ spaced time modulated linear array with the same number of elements, the obtained value of SLL of the synthesized patterns is $-20 \mathrm{~dB}$. In the proposed approach, using the element position in combination with modulation on-time duration as optimization parameter, reduction of SLLs of PB and FTB are improved by $4.6 \mathrm{~dB}$ and $4.75 \mathrm{~dB}$, respectively, as compared to those obtained in [25]. In [29], though the SLLs of the synthesized patterns in unequally spaced CPA are reduced more, however, higher DRR (=10.29) of static excitation amplitudes leads to increase of the design cost and complexity of the practical array. Although uniform amplitude excitation can be used in the multiple-pattern synthesis in CPA [30], higher SLLs of the synthesized patterns make them unsuitable for some applications. Also for the conventional arrays as considered in [31], [32], a pre-defined amplitude excitation with relatively higher dynamic range ratios (DRR) as mentioned in Tab. 1 is required to realize the desired patterns. On the 
other hand, using the proposed method desired low SLL patterns can be achieved only by controlling the on-off status of the RF switches with their respective OTDs with the help of software. Thus the hardware implementation of the proposed method is much easier than a CPA one with higher DRR.

\subsection{Case 2: Synthesis of Sum and Difference Patterns Controlled by OTDs and OTIs of the Switches}

In this case, a TMLA of 30 isotropic elements is considered for the synthesis of sum and difference patterns. The array is symmetric at the origin of the co-ordinate axis. The excitation amplitude distribution of the TMLA is prefixed as uniform, leaving the on-time duration (OTD) and on-time instants (OTI) different for each pattern. The pulse shifting strategy as detailed in [20] is applied to synthesize the desired patterns. The cost function as defined in (10) is minimized using DE. Thus to generate desired radiation pattern the OTDs, OTIs and element positions are considered as optimization parameter vector for DE. Here, the main objective is to find out a common set of element position for the desired dual patterns while different sets of OTDs and OTIs are responsible to shape the pattern in sum and difference form.

The search range of the optimization parameter vectors are defined as: element spacing $[0.5,1]$, normalized OTDs $[0.1,1]$ and OTIs $[0,(1-$ OTD $)]$ so that summation of them becomes less than or equal to 1 . For sum and difference patterns, the desired values of SLL, SBL and FNBW in the cost function are set as $S L L_{\mathrm{d}}=-25 \mathrm{~dB}$, $S B L_{\mathrm{d}}=-30 \mathrm{~dB}$ and $F N B W_{\mathrm{d}}=10^{\circ}$. The synthesized sum and difference pattern are shown in Fig. 6. From the DE optimized sum pattern the obtained values of SLL, SBL and FNBW are found as $-24.8 \mathrm{~dB},-26.5 \mathrm{~dB}$ and $8.6^{\circ}$. Similarly for difference pattern the obtained values of SLL, SBL and FNBW are $-22.8 \mathrm{~dB},-25.8 \mathrm{~dB}$ and $8.8^{\circ}$, respectively. The optimum solutions consisting of common element positions and different set of OTDs and OTIs are

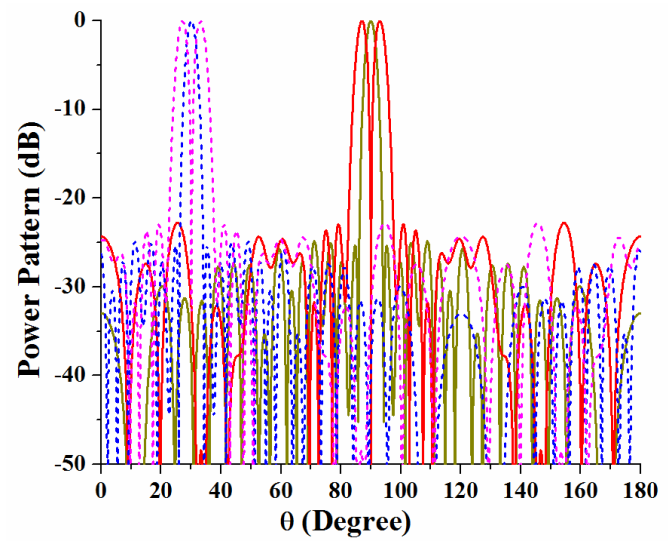

Fig. 6. DE optimized sum and difference pattern for 30 element linear array where mainlobe is at $\theta=30^{\circ}$ and $\theta=90^{\circ}$.

\begin{tabular}{|c|c|c|c|c|c|}
\hline \multirow{2}{*}{$\begin{array}{c}\text { Element } \\
\text { number }\end{array}$} & $\begin{array}{c}\text { Element } \\
\text { position } \boldsymbol{x}_{\boldsymbol{n}}\end{array}$ & \multicolumn{2}{c|}{ Sum pattern } & \multicolumn{2}{c|}{$\begin{array}{c}\text { Difference } \\
\text { pattern }\end{array}$} \\
\cline { 3 - 6 } & in terms of $\lambda$ & OTD & OTI & OTD & OTI \\
\hline \pm 1 & \pm 0.25 & 0.34 & 0.62 & 0 & 0.91 \\
\hline \pm 2 & \pm 0.75 & 0.63 & 0.34 & 0 & 0.99 \\
\hline \pm 3 & \pm 1.25 & 0.37 & 0.02 & 0.36 & 0.55 \\
\hline \pm 4 & \pm 1.86 & 0.85 & 0.14 & 0.77 & 0.10 \\
\hline \pm 5 & \pm 2.47 & 0.79 & 0 & 0.99 & 0.008 \\
\hline \pm 6 & \pm 2.99 & 0.90 & 0.08 & 0.99 & 0.0002 \\
\hline \pm 7 & \pm 3.49 & 0.90 & 0.07 & 0.99 & 0 \\
\hline \pm 8 & \pm 4.02 & 0.95 & 0.02 & 0.98 & 0.006 \\
\hline \pm 9 & \pm 4.57 & 0.95 & 0.01 & 0.99 & 0.002 \\
\hline \pm 10 & \pm 5.26 & 1 & 0 & 0.99 & 0.007 \\
\hline \pm 11 & \pm 6.05 & 0.99 & 0.004 & 0.99 & 0.001 \\
\hline \pm 12 & \pm 6.88 & 0.99 & 0.001 & 0.99 & 0.003 \\
\hline \pm 13 & \pm 7.73 & 0.99 & 0.004 & 0.90 & 0.083 \\
\hline \pm 14 & \pm 8.60 & 0.99 & 0.0001 & 0.07 & 0.084 \\
\hline \pm 15 & \pm 9.46 & 0.99 & 0.0048 & 0.08 & 0.0002 \\
\hline
\end{tabular}

Tab. 2. Element-wise optimization parameter values for sum and difference pattern obtained using DE algorithm.

presented in Tab. 2. In comparison with [25], approximately $4.8 \mathrm{~dB}$ improvement in SLL is observed in this case. As in [33], almost the same pattern is obtained in conventional antennas by using non-uniform excitation amplitude distribution with DRR of 5. However, in this proposed approach, the desired low SLL patterns can be achieved only by controlling the on-off sequence of the RF switches with their respective OTDs and OTIs. Therefore, the hardware implementation of this approach is much easier than conventional antenna arrays.

\subsection{Case 3: Synthesis of Sum and Difference Patterns Using Phase Only Control}

In this case, a TMLA of 30 isotropic elements is considered for synthesis of sum and difference pattern using phase-only control having uniform excitation amplitude distribution. The tuning parameter values of $\mathrm{DE}$ are same as considered in case 1 . The main objective is to optimize the sum and difference patterns with variation in phase while maintaining common element position, excitation am-

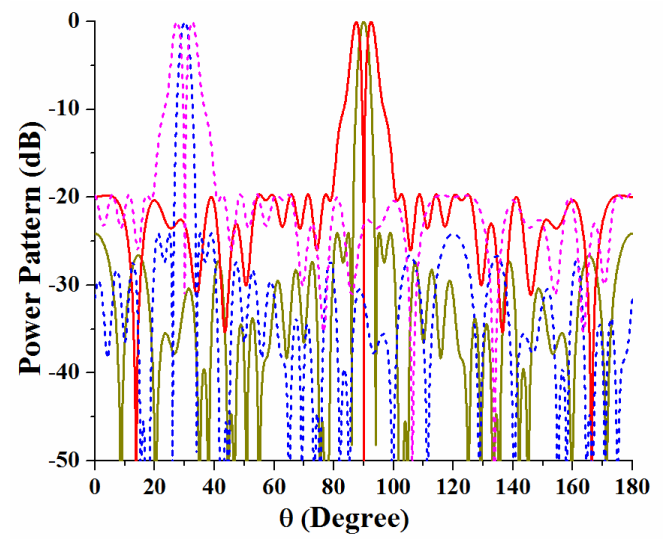

Fig. 7. DE optimized sum and difference pattern for 30 element linear array by phase only synthesis where mainlobe is at $\theta=30^{\circ}$ and $\theta=90^{\circ}$. 


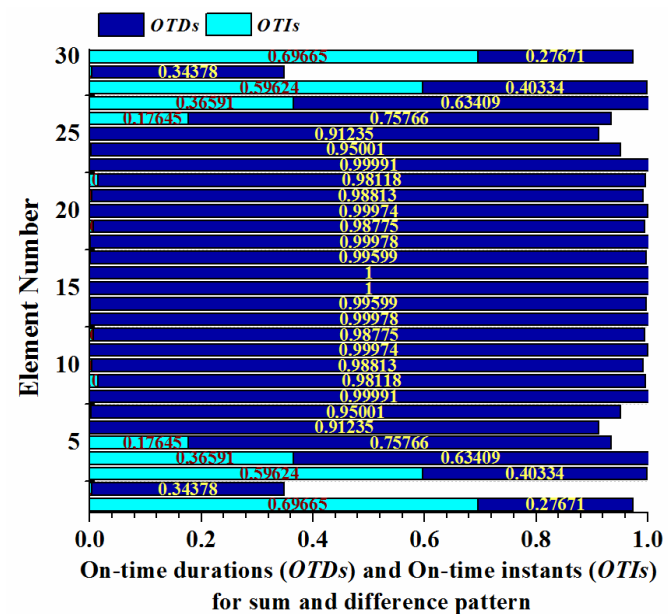

Fig. 8. DE optimized On-time duration and On-time instants for sum and difference pattern.

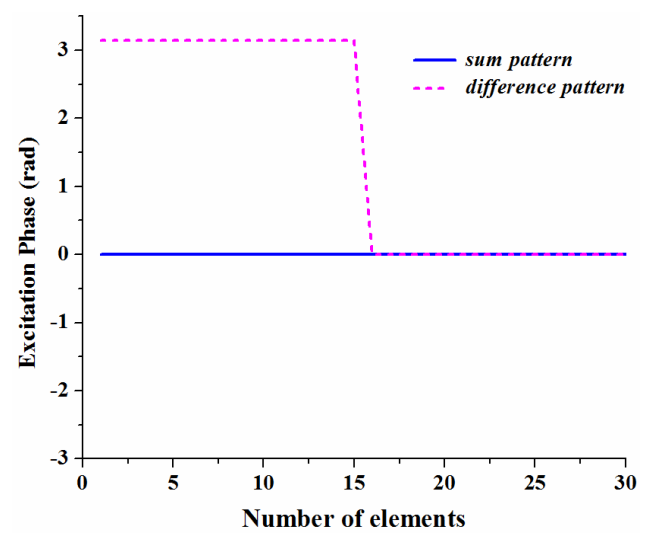

Fig. 9. DE optimized excitation phase for sum and difference pattern.

plitude and switch-on time durations. In this case, the desired value of SLL, SBL and FNBW are considered as $-25 \mathrm{~dB},-30 \mathrm{~dB}$ and $20^{\circ}$, respectively. The DE optimized sum and difference pattern is shown in Fig. 7 where the obtained values of SLL, SBL and FNBW are $-24.07 \mathrm{~dB}$, $-26.45 \mathrm{~dB}$ and $8^{\circ}$ for sum pattern and for difference pattern the obtained values of SLL, SBL and FNBW are observed as $-19.65 \mathrm{~dB},-22.98 \mathrm{~dB}$ and $11.4^{\circ}$, respectively.

The DE optimized OTDs, OTIs and excitation phase are shown in Fig. 8 and 9. In the proposed approach reduction of SLLs are improved by $8 \mathrm{~dB}$, as compared to those obtained in [30]. In comparison to [31] almost the same pattern is observed in conventional antenna array with equal element spacing. This implies the result obtained in this case is the optimum one.

\subsection{Case 4: Synthesis of Sum and Difference Patterns with Less Number of Time- Modulating Elements}

Since for a particular pattern, the predetermined OTDs and OTIs are to be established through the feed network of the array. Thus, switching from one array pattern to another requires appropriate control of the ON-OFF status of the switches as per the corresponding OTDs and OTIs of the pattern. Hence, during switching from one pattern to another, controlling more number of switches increases the complexity in the feeding network. In this case, with the aim of reducing the complexity in the feed network, the desired sum and difference pattern is synthesized not only with a common element position but also with a percentage of elements having common excitation. For the individual pattern, time-modulation is applied to few elements only. Therefore, generation of dual-beam patterns with a higher sharing percentage in element excitations results in lower complexity and cost. The normalized power patterns obtained by DE algorithm are shown in Fig. 10.

Table 3 lists the element position with uniform excitation and on-time durations for sum and difference pattern obtained after optimization using DE. For sum pattern, the obtained values of $S L L=-23.44 \mathrm{~dB}, S B L=-27.83 \mathrm{~dB}$ and $F N B W=8.8^{\circ}$, and for difference pattern the obtained values are $S L L=-21.32 \mathrm{~dB}, S B L=-22.71 \mathrm{~dB}$ and $F N B W=8^{\circ}$.

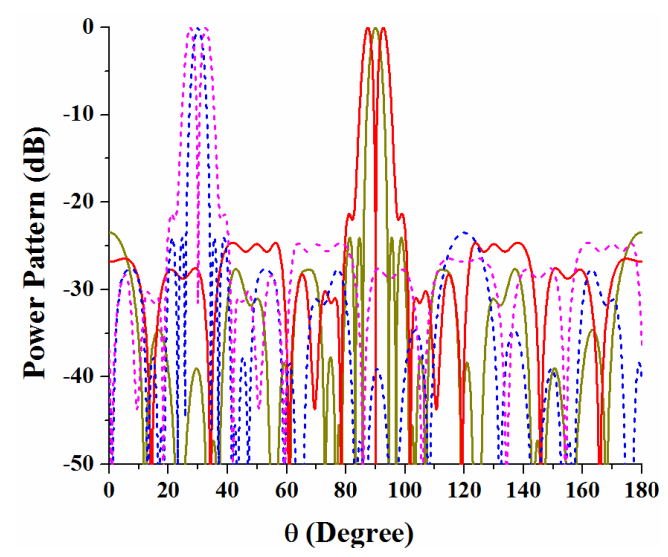

Fig. 10. Sum and difference pattern obtained using optimized element excitation where mainlobe is at $\theta=30^{\circ}$ and $\theta=90^{\circ}$.

\begin{tabular}{|c|c|c|c|c|}
\hline \multirow{2}{*}{$\begin{array}{l}\text { Element } \\
\text { number }\end{array}$} & \multirow{2}{*}{$\begin{array}{c}\text { Element } \\
\text { position } \\
\left(x_{n}\right) \text { in } \\
\text { terms of } \lambda\end{array}$} & \multirow{2}{*}{$\begin{array}{l}\text { Uniform } \\
\text { static } \\
\text { excitation }\end{array}$} & \multicolumn{2}{|c|}{ Normalized OTDs } \\
\hline & & & $\begin{array}{c}\text { Sum } \\
\text { pattern }\end{array}$ & $\begin{array}{c}\text { Difference } \\
\text { pattern }\end{array}$ \\
\hline \pm 1 & \pm 0.26 & 1 & 0.5000 & 1 \\
\hline \pm 2 & \pm 0.84 & 1 & 0.5706 & 1 \\
\hline \pm 3 & \pm 1.34 & 1 & 0.5006 & 1 \\
\hline \pm 4 & \pm 1.84 & 1 & 1 & 1 \\
\hline \pm 5 & \pm 2.55 & 1 & 1 & 1 \\
\hline \pm 6 & \pm 3.25 & 1 & 1 & 1 \\
\hline \pm 7 & \pm 3.91 & 1 & 1 & 1 \\
\hline \pm 8 & \pm 4.61 & 1 & 1 & 1 \\
\hline \pm 9 & \pm 5.46 & 1 & 1 & 1 \\
\hline \pm 10 & \pm 6.31 & 1 & 1 & 1 \\
\hline \pm 11 & \pm 7.16 & 1 & 1 & 1 \\
\hline \pm 12 & \pm 8.00 & 1 & 1 & 1 \\
\hline \pm 13 & \pm 8.51 & 1 & 1 & 0.2000 \\
\hline \pm 14 & \pm 9.01 & 1 & 1 & 0.3129 \\
\hline \pm 15 & \pm 9.81 & 1 & 1 & 0.2010 \\
\hline
\end{tabular}

Tab. 3. Element-wise uniform excitations and OTDs for sum and difference pattern obtained using DE algorithm. 


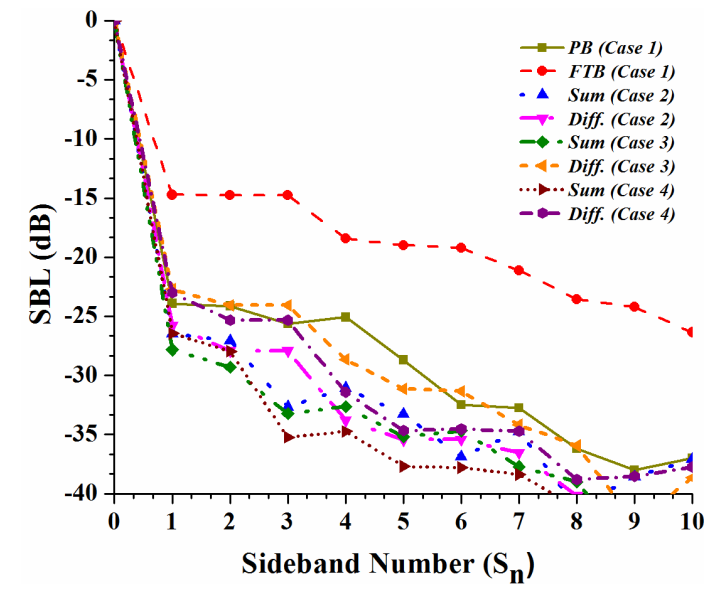

Fig. 11. Sideband level at first 10 sidebands obtained from different optimization methods.

As compared with [24], approximately $7.6 \mathrm{~dB}$ improvement in SLL for sum pattern and $4.9 \mathrm{~dB}$ improvement in SLL for difference pattern is observed in this case. Also, to obtain the difference pattern in [24], four elements are to be time-modulated while in the proposed method, improved performance is observed with time-modulation of 3 elements. For the synthesized patterns as considered under case 1 to 4 , the maximum SBL of first 10 sidebands are presented in Fig. 11. It can be seen that the SBL at higher order sidebands are suppressed significantly. From the results illustrated under different cases, it can be observed that using the proposed method, without changing the element position the desired dual-beam patterns can be realized only by altering the ON-OFF time sequence of the RF switches. However, if multiple patterns are synthesized using the method reported in [26], element position will be different for the different patterns and a complex feed network will be required to achieve the patterns. To show the suitability of the proposed method in the application for estimating the direction of arrival (DoA), all the synthesized patterns are steered along $30^{\circ}$ as depicted with dotted lines along with the synthesized broadside patterns in Fig. 4, 6, 7 and 10. Almost the same pattern characteristics at the steering angle show the applicability of the designed array for the wide angular range. This indicates the superiority and effectiveness of the proposed approach in terms of realizing low SLL patterns as compared to the previously published works.

\section{Conclusion}

This paper focusses in improving the SLL, SBL of multiple radiation patterns by applying time modulation in unequally spaced linear array with the help of DE. The technique represents an up-gradation for re-configurability purpose. Numerical results corresponding to different radiation patterns including the sum, difference and flat-topped pattern synthesized from time modulated unequally spaced linear array are compared with conventional arrays. After comparison with some of the published articles, it is found that multiple patterns obtained from TMLAs have much lower side lobes compared to conventional antenna arrays. Moreover, the study shows that unequally spaced linear arrays with pre-fixed amplitude distribution can be utilized to synthesize multiple patterns. The SBL reduction and minimization of SBR have been observed. Finally, this method can also be extended further to synthesize circular, planar array antennas.

\section{Acknowledgement}

This work is under the DST-SERB project Ref. file number EEQ/2016/00836, dated January 17, 2017.

\section{References}

[1] BARBA, M., PAGE, J. E., ENCINAR, J. A., et al. A switchable multiple beam antenna for GSM-UMTS base stations in planar technology. IEEE Transactions on Antennas and Propagation, 2006, vol. 54, no. 11, p. 3087-3094. DOI: 10.1109/TAP.2006.883991

[2] LAGER, I. E., TRAMPUZ, C., SIMEONI, M., et al. Interleaved array antennas for FMCW radar applications. IEEE Transactions on Antennas and Propagation, 2009, vol. 57, no. 8, p. 2486-2490. DOI: 10.1109/TAP.2009.2024573

[3] WU, L., ZIELINSKI, A., BIRD, J. S. Synthesis of shaped radiation patterns using an iterative method. Radio Science, 1995, vol. 30, no. 5, p. 1385-1392. DOI: 10.1029/95RS01829

[4] BUCKLEY, M. J. Synthesis of shaped beam antenna patterns using implicitly constrained current elements. IEEE Transactions on Antennas and Propagation, 1996, vol. 44, no. 2, p. 192-197. DOI: $10.1109 / 8.481647$

[5] HAUPT, R. L. Phase-only adaptive nulling with a genetic algorithm. IEEE Transactions on Antennas and Propagation, 1997, vol. 45, no. 6, p. 1009-1015. DOI: 10.1109/8.585749

[6] DIAZ, X., RODRIGUEZ, J. A., ARES, F., et al. Design of phase-differentiated multiple-pattern antenna arrays. Microwave and Optical Technology Letters, 2000, vol. 26, p. 52-53. DOI: 10.1002/(SICI)1098-2760(20000705)26:1<52::AIDMOP16>3.0.CO;2-0

[7] KUMMER, W., VILLENEUVE, A., FONG, T., et al. Ultra-low sidelobes from time-modulated arrays. IEEE Transactions on Antennas and Propagation, 1963, vol. 11, no. 6, p. 633-639. DOI: 10.1109/TAP.1963.1138102

[8] ZHOU, H. J., SUN, B. H., LI, J. F., et al. Efficient optimization and realization of a shaped-beam planar array for very large array application. Progress In Electromagnetics Research, 2009, vol. 89, p. 1-10. DOI: 10.2528/PIER08112503

[9] SHANKS, H. E., BICKMORE, R. W. Four-dimensional electromagnetic radiators. Canadian Journal of Physics, 1959, vol. 37 , no. 3 , p. $263-275$. DOI: $10.1139 /$ p59-031

[10] BALANIS, C. A. Antenna Theory: Analysis and Design. Hoboken (NJ, USA): Wiley, 2005. ISBN: 978-1-118-64206-1

[11] LEBRET, H., BOYD, S. Antenna array pattern synthesis via convex optimization. IEEE Transactions on Signal Processing, 1997, vol. 45 , no. 3 , p. 526-532. DOI: $10.1109 / 78.558465$

[12] YANG, Y., LIU, Y., MA, X., et al. Synthesizing unequally spaced pattern-reconfigurable linear arrays with minimum interspacing control. IEEE Access, 2019, vol. 7, p. 58893-58900. DOI: 10.1109/ACCESS.2019.2914767 
[13] YANG, K., ZHAO, Z., LIU, Q. H. Fast pencil beam pattern synthesis of large unequally spaced antenna arrays. IEEE Transactions on Antennas and Propagation, 2013, vol. 61, no. 2, p. 627-634. DOI: 10.1109/TAP.2012.2220319

[14] YANG, S., GAN, Y. B., QING, A. Sideband suppression in timemodulated linear arrays by the differential evolution algorithm. IEEE Antennas and Wireless Propagation Letters, 2002, vol. 1, p. 173-175.DOI: 10.1109/LAWP.2002.807789

[15] MANDAL, S. K., MAHANTI, G., GHATAK, R. Differential evolution algorithm for optimizing the conflicting parameters in time-modulated linear array antennas. Progress In Electromagnetics Research B, 2013, vol. 51, p. 101-118. DOI: 10.2528/PIERB13022710

[16] FONDEVILA, J., BREGAINS, J. C., ARES, F., et al. Optimizing uniformly excited linear arrays through time modulation. IEEE Antennas and Wireless Propagation Letters, 2004, vol. 3, p. 298-301. DOI: 10.1109/LAWP.2004.838833

[17] YANG, S., GAN, Y. B., QING, A., et al. Design of a uniform amplitude time modulated linear array with optimized time sequences. IEEE Transactions on Antennas and Propagation, 2005, vol. 53 , no. 7, p. 2337-2339. DOI: 10.1109/TAP.2005.850765

[18] POLI, L., ROCCA, P., OLIVERI, G., et al. Harmonic beamforming in time-modulated linear arrays. IEEE Transactions on Antennas and Propagation, 2011, vol. 59, no. 7, p. 2538-2545. DOI: 10.1109/TAP.2011.2152323

[19] POLI, L., ROCCA, P., MANICA, L., et al. Pattern synthesis in time-modulated linear arrays through pulse shifting. IET Microwaves, Antennas and Propagation, 2010, vol. 4, no. 9, p. 1157-1164. DOI: 10.1049/iet-map.2009.0042

[20] BEKELE, E. T., POLI, L., ROCCA, P., et al. Pulse-shaping strategy for time modulated arrays-Analysis and design. IEEE Transactions on Antennas and Propagation, 2013, vol. 61, no. 7, p. 3525-3537. DOI: 10.1109/TAP.2013.2256096

[21] YANG, S., LI, G., HUANG, M., et al. Shaped patterns synthesis in time-modulated antenna arrays with static uniform amplitude and phase excitations. Frontiers of Electrical and Electronic Engineering in China, 2010, vol. 5, no. 2, p. 179-184. DOI: $10.1007 / \mathrm{s} 11460-010-0005-2$

[22] PATRA, S., MANDAL, S. K., MAHANTI, G. K., et al. Synthesis of flat-top power pattern in time-modulated unequally spaced linear arrays using DE. In IEEE 2nd International Conference on Recent Trends in Information Systems (ReTIS). Kolkata (India), 2015, p. 104-108. DOI: 10.1109/ReTIS.2015.7232861

[23] MANDAL, S. K., MAHANTI, G., GHATAK, R. Synthesis of simultaneous multiple-harmonic-patterns in time-modulated linear antenna arrays. Progress In Electromagnetics Research M, 2014 vol. 34, p. 135-142. DOI: 10.2528/PIERM13111802

[24] FONDEVILA, J., BREGAINS, J. C., ARES, F., et al. Application of time modulation in the synthesis of sum and difference patterns by using linear arrays. Microwave and Optical Technology Letters, 2006, vol. 48, no. 5, p. 829-832.DOI: 10.1002/mop.21489

[25] YANG, S., CHEN, Y., NIE, Z. Multiple patterns from timemodulated linear antenna arrays. Electromagnetics, 2008, vol. 28, no. 8, p. 562-571. DOI: 10.1080/02726340802428671

[26] LI, G., YANG, S., HUANG, M., et al. Sidelobe suppression in time modulated linear arrays with unequal element spacing. Journal of Electromagnetic Waves and Applications, 2012, vol. 24, no. 5-6, p. 775-783. DOI: $10.1163 / 156939310791036368$

[27] BREGAINS, J. C., FONDEVILA-GOMEZ, J., FRANCESCHETTI, G., et al. Signal radiation and power losses of time-modulated arrays. IEEE Transactions on Antennas and Propagation, 2008, vol. 56, no. 6, p. 1799-1804. DOI: 10.1109/TAP.2008.923345

[28] ROCCA, P., OLIVERI, G., MASSA, A. Differential evolution as applied to electromagnetics. IEEE Antennas and Propagation Magazine, 2011, vol. 53, no. 1, p. 38-49. DOI: 10.1109/MAP.2011.5773566
[29] VAITHEESWARAN, S. M. Dual beam synthesis using element position perturbations and the G3-GA algorithm. Progress In Electromagnetics Research, 2008, vol. 87, p. 43-61. DOI: 10.2528/PIER08091601

[30] DURR, M., TRASTOY, A., ARES, F. Multiple-pattern linear antenna arrays with single prefixed amplitude distributions: Modified Woodward-Lawson synthesis. Electronics Letters, 2000, vol. 36, no. 16, p. 1345-1346. DOI: 10.1049/el:20000980

[31] MAHANTI, G., CHAKRABORTY, A., DAS, S. Phase-only and amplitude-phase only synthesis of dual-beam pattern linear antenna arrays using floating-point genetic algorithms. Progress In Electromagnetics Research, 2007, vol. 68, p. 247-259. DOI: 10.2528/PIER06072301

[32] BUCCI, O. M., MAZZARELlA, G., PANARIELlO, G. Reconfigurable arrays by phase-only control. IEEE Transactions on Antennas and Propagation, 1991, vol. 39, no. 7, p. 919-925. DOI: $10.1109 / 8.86910$

[33] MOHAMMED, J. R. Synthesizing sum and difference patterns with low complexity feeding network by sharing element excitations. International Journal of Antennas and Propagation, 2017, vol. 2017, p. 1-7. DOI: $10.1155 / 2017 / 2563901$

\section{About the Authors ...}

Somnath PATRA received B-Tech \& M-Tech degree from WBUT in 2008 \& 2011 respectively. He is currently pursuing his Ph.D. from NIT Durgapur, India. He has more than 8 years of teaching experience. He has published research papers in national and international peer reviewed journals and conferences.

Sujit Kumar MANDAL received B.Sc. degree in Physics (H) from the University of Calcutta in 2001. He completed B.Tech. and M.Tech. in Radio Physics \& Electronics from C. U. in the year 2004 and 2006, respectively. He received Ph.D. degree in the year 2014 from NIT, Durgapur. Presently, he is working as Assistant Professor in the Dept. of ECE, NIT Durgapur, India. He has published more than 50 research papers in national and international peer reviewed journals and conferences. His research area includes application of soft computing techniques in antenna array optimization, time-modulated antenna arrays, microstrip patch antenna, RF energy harvesting and on-chip antenna design.

Gautam Kumar MAHANTI received B.E. in ECE from NIT Durgapur in 1988, M.E. in Electronics System and Communication from NIT, Rourkela in 1994 and Ph.D. from IIT, Kharagpur, India. He has more than 20 years of teaching and research experience. Presently, he is working as Professor at the Dept. of Electronics and Communication Engineering, National Inst. of Technology, Durgapur, India. He is a senior member of IEEE, USA. He has published approx. 70 papers in journals and in national and international conferences. He was the reviewer of many international journals such as Electronics Letter, IEEE Antennas and Wireless Propagation Letter, Progress in Electromagnetics Research, International Journal of Adaptive Control and Signal Processing, and many conferences.

Narendra Nath PATHAK received his Ph.D. degree from NIT Durgapur and is now working as Professor and HOD in Dr. B. C. Roy Engineering College, Durgapur, India. 